$(58.3 \%)$ patients deceased and $2(16.7 \%)$ were alive without evidence of cancer.

Conclusion* in our series, bevacizumab had the expected outcomes and safety profile. Nevertheless, multicentric studies are needed to evaluate the true added value of bevacizumab to P-CT in ACC treatment in real-world practice and to identify predictive factors.

\section{EFFICACY OF CHECKPOINT INHIBITOR IMMUNOTHERAPY DRUG PEMBROLIZUMAB (KEYTRUDA) FOR TREATMENT OF ADVANCED CERVICAL CANCER}

${ }^{1} \mathrm{~F}$ Ahmed, ${ }^{2} \mathrm{Z}$ Shah*, ${ }^{1} \mathrm{I}$ Khan, ${ }^{1} \mathrm{~A}$ Bhatnagar, ${ }^{1} \mathrm{R}$ Garimella, ${ }^{1} \mathrm{~S}$ Mishra, ${ }^{3} \mathrm{~S}$ Gudapati, ${ }^{3} \mathrm{SH}$ Bobba, ${ }^{4} \mathrm{~S}$ Zaidi, ${ }^{5} \mathrm{~K}$ Andrews, ${ }^{6} \mathrm{U}$ Salman, ${ }^{1} \mathrm{Z}$ Yukselen, ${ }^{1} \mathrm{P}$ Guntipalli, ${ }^{7} \mathrm{R}$ Zarrar, ${ }^{8} \mathrm{G}$ Enebong Nya, ${ }^{9} \mathrm{~S}$ Ahmad. ${ }^{1}$ Larkin Community Hospital, Division of Clinical and Translational Research, South Miami, USA; ${ }^{2}$ Weiss Memorial Hospital, Department of Internal Medicine, Chicago, USA; ${ }^{3}$ Washington University of Health and Science, College of Medicine, San Pedro, Belize; 'Lahore Medical and Dental College, College of Medicine, Lahore, Pakistan; ${ }^{5}$ Prince Mohammad Bin Fahad University, Department of CORE, Al Khobar, Saudi Arabia; ${ }^{6}$ Bakhtawar Amin Medical and Dental College, College of Medicine, Multan, Pakistan; ' Mayo Clinic, Department of Oncology, Rochester, USA; ${ }^{8}$ John Hopkins Hospital, Department of Gynecologic Oncology, Baltimore, USA; ${ }^{9}$ Advent Health Cancer Institute, Division of Gynecologic Oncology, Orlando, USA

\subsection{6/ijgc-2021-ESG0.43}

Introduction/Background* Cervical cancer is the fourth most common cause of cancer-related deaths in women worldwide. With screening for precancerous lesions and vaccination for preventive human papillomavirus (HPV), a survival improvement has been observed in these patients in developed countries. In recent years, immunotherapy has represented a breakthrough in oncology and immune-checkpoint inhibitors have shown antitumor activity in a variety of tumor types. Here, we present the first systematic review discussing the efficacy and clinical usefulness of pembrolizumab, an anti-PD1 checkpoint inhibitor, in the treatment of advanced cervical cancer.

Methodology A systematic literature search was performed on March 2021 according to PRISMA statement using multiple databases and selective medical search terms (MeSH) such as 'uterine cervical neoplasms' AND 'Pembrolizumab'. After a detailed primary and secondary screening conducted by two members of 188 studies, total 7 studies were included (Chung et al. 2019, Frenel et al. 2017, Choi, M et al. 2020, H Chung et al. 2018, SH Kim al. 2019, J Zhu et al.2019, K. Miller et al. 2020).

Result(s)* A total of 411 patients, mean age of 48 years (2176) with advanced cervical cancer who had received a median range of 1-7 previous lines of therapies, were included. In all studies, pembrolizumab monotherapy reported a cumulative median duration of follow up of 10.5 months (0-32.2), overall response rate $(\mathrm{ORR})$ of $15 \% \quad(\mathrm{n}=47 / 313)$, and complete response of $3.76 \%(n=14 / 372)$. Very Good partial response was not reported in any study. A calculated pooled partial response, stable disease, and progressive disease were reported respectively $[9.7 \%(n=40 / 411)$ vs. $19.3 \%(n=67 / 346)$ vs. $41 \%$ $(n=145 / 348)]$. Six-month overall survival as presented in 3 studies was $67 \%(n=160 / 239)$. The progression free survival and survival data is premature at this stage and requires further elaboration in phase II/III clinical trials.

Conclusion* Pembrolizumab (KEYTRUDA) monotherapy demonstrated durable antitumor activity in patients with advanced cervical cancer. However, further studies using combinations with other treatment options including chemotherapy, radiotherapy and other immunotherapeutic agents should be explored for the efficacy and survival outcomes.

\section{CERVICAL CANCER AFTER LACC TRIAL...WHAT DO WE DO?}

C Darin*, JDI Guilmi, J Quiroga Luna, G Maya. british hospital or buenos aires, gynecology oncology, caba, Argentina

\subsection{6/ijgc-2021-ESG0.44}

Introduction/Background* In 2018 the results of an early termination of the LACC trial were published, followed by a tremendous amount of retrospective trials, all showing the worst outcomes of patients with cervical cancer who were operated by minimally invasive surgery (MIS). After years of training in laparoscopic radical hysterectomy we had go backwards and start learning the open way once again

Methodology Educational video showing new strategies in patients with cervical cancer after LACC trial

Result(s)* Since we already have the laparoscopic platform for sentinel lymph node (SLN) mapping with Indocyanine green (ICG), we decided we will start by doing our surgeries with MIS. After cervical injection of ICG, we search for bilateral SLN. The radical hysterectomy is prepared through laparoscopy by dissecting the retroperitoneal space, identifying vascular structures and ureter. The procedure is continued by the open way. We Coagulate and section uterine artery and ventral, lateral, and posterior parametrium. For the colpectomy we used bigger vaginal clamps in order to avoid tumour spillage inside the abdominal cavity.

In 2019 al 2020 Dr Kohler and Dr Chiva suggested that closing the vagina over the tumour, is a feasible technique that could avoid tumour spillage and may improve the outcomes in MIS. So, we began our learning curve in vaginal cuff in open surgery. After the laparoscopic SLN mapping, we have a vaginal time, and finally the open surgery.

Conclusion* Prospective randomized trials are needed to prove that MIS is safe for our patients with the adding of surgical changes as the vaginal cuff. Meanwhile we keep doing open surgeries with some advantages such as the laparoscopic SNL mapping and the spaces dissection, decreasing the open operating time, and the possibility of tumour spillage with the vaginal cuff.

\section{LONG TERM TREATMENT OUTCOME AFTER PELVIC EXENTERATIONS FOR RECURRENT GYNAECOLOGICAL CANCERS}

${ }^{1}$ PN Rema* , 'S Sambasivan, ${ }^{2} \mathrm{~A}$ Nath, ${ }^{2} \mathrm{~S}$ Ranjith J, ${ }^{3} \mathrm{~A}$ Mathew. ${ }^{1}$ Regional cancer center, Department of Gynaecologicaloncology, Thiruvananthapuram, India; ${ }^{2}$ Regional cancer center, Department of surgical oncology, Thiruvananthapuram, India; ${ }^{3}$ Regional cancer center, Department of Epidemiology and biostatistics, Thiruvananthapuram, India

\subsection{6/ijgc-2021-ESGO.45}

Introduction/Background* Pelvic exenteration is radical enbloc resection of pelvic organs followed by surgical reconstruction. This is considered a curative treatment in recurrent gynaecological cancers. The present study looked into the long term survival outcome and morbidities associated with pelvic exenterations done in our centre. 